\title{
Nanoscale
}

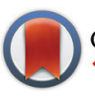

CrossMark $\leftarrow$ click for updates

Cite this: Nanoscale, 2016, 8, 18098

Received 21st July 2016

Accepted 6th October 2016

DOI: 10.1039/c6nr05767e

www.rsc.org/nanoscale

\section{Precise redox-sensitive cleavage sites for improved bioactivity of siRNA lipopolyplexes $\uparrow$}

\author{
Philipp Michael Klein, $\ddagger^{a}$ Sören Reinhard, $\ddagger^{a}$ Dian-Jang Lee, ${ }^{a, b}$ Katharina Müller, ${ }^{a}$ \\ Daniela Ponader, ${ }^{c}$ Laura Hartmann ${ }^{d}$ and Ernst Wagner ${ }^{\star a, b}$
}

\begin{abstract}
Lipo-oligomers have been proven as potent siRNA carriers based on stable electrostatic and hydrophobic complex formation and endosomal membrane destabilization. Although high stability of siRNA polyplexes is desirable in the extracellular space and cellular uptake, intracellular disassembly is important for the cytosolic release of siRNA and RNA-induced silencing complex formation. To improve the release, bioreducible sequence-defined lipo-oligomers were synthesized by solid-phase assisted synthesis using the disulfide building block Fmoc-succinoyl-cystamine for precise positioning of a disulfide unit between a lipophilic diacyl (bis-myristyl, bis-stearyl or bis-cholestanyl) domain and an ionizable oligocationic siRNA binding unit. Reducible siRNA polyplexes show higher gene silencing efficacy and lower cytotoxicity than their stable analogs, consistent with glutathione-triggered siRNA release and reduced lytic activity.
\end{abstract}

\section{Introduction}

RNA interference (RNAi) initiated by cytosolic delivery of synthetic small interfering RNA (siRNA) provides innovative therapeutic opportunities for the regulation of disease-associated genes. ${ }^{1,2}$ A dynamic and bioresponsive design of the delivery system is essential to overcome various extracellular and intracellular hurdles. ${ }^{3-12}$ Driven by electrostatic interaction with negatively charged nucleic acids and intermolecular hydrophobic association, cationic lipids or lipo-oligomers assemble into lipopolyplexes. This type of carrier stands out due to high siRNA complex stability and transfection efficiency. ${ }^{13-15}$ Establishment of clear structure-activity relationships for further optimization depends on availability of polymeric carriers with precise chemical structure. ${ }^{16,17}$ Solid-phase supported synthesis (SPS) is one approach for accurate assembly in a defined way. ${ }^{18-25}$ Combining natural with artificial amino acids and other synthetic building blocks, oligomers with specific topologies and different functional elements can be

\footnotetext{
${ }^{a}$ Pharmaceutical Biotechnology, Department of Pharmacy, Center of Nanoscience (CeNS), Ludwig-Maximilians-Universität, Butenandtstr. 5-13, 81377 München, Germany.E-mail: ernst.wagner@cup.uni-muenchen.de

${ }^{b}$ Nanosystems Initiative Munich (NIM), Schellingstr. 4, 80799 München, Germany

${ }^{c}$ Max-Planck-Institute of Colloids and Interfaces, Department of Biomolecular Systems, Am Mühlenberg 1, 14476 Potsdam-Golm, Germany

${ }^{d}$ Institut für Organische Chemie und Makromolekulare Chemie, Heinrich-HeineUniversität Düsseldorf, Universitätsstr. 1, 40225 Düsseldorf, Germany.

E-mail: laura.hartmann@hhu.de

$\dagger$ Electronic supplementary information (ESI) available. See DOI: 10.1039/ c6nr05767e

$\$$ These authors contributed equally.
}

designed; our previous work demonstrated the beneficial effect of hydrophobic elements and disulfide-forming cysteines on siRNA polyplex stabilization. ${ }^{26-28}$ Maximum stabilization, however, was not advantageous for gene silencing; the incorporation of disulfide bonds should facilitate disassembly of polyplexes in the intracellular reductive milieu caused by

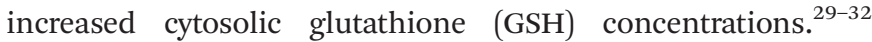
Cysteine-based disulfide formation during polyplex formation by air oxygen is a poorly controllable, incomplete process. ${ }^{21,24}$ Alternatively, integration of bioreducible bonds into carriers can be achieved before nanoparticle formation by polymerization reactions with disulfide-bearing compounds. ${ }^{30-32}$ The positioning of the bioreducible element can to some extend be controlled via sequential assembly of the carrier. Disulfide bonds can be more accurately integrated during SPS of polymers, as already demonstrated by Hartmann et al. ${ }^{33}$ Cleavable cationic domains as well as disassembly of stabilizing domains have been demonstrated to improve delivery systems. ${ }^{29}$

In the current work, we designed novel bioreducible cationic lipo-oligomers. By precisely positioning the disulfide bond between the fatty acid and polycationic domain (and not into the polycationic domain) via a Fmoc-succinoyl-cystamine building block, we intended to obtain a most drastic molecular change upon bioreduction. The amphiphilic, detergent-like character, which is considered favorable for endosomolysis but might also be associated with cytotoxicity, should be abolished upon entry into the reductive cytosol by the split into separate pure lipidic and cationic fragments; the latter fragments have insufficient ability to bind siRNA.

In this study, we evaluate three lipo-oligomer topologies (T-shape, i-shape and U-shape) and different representatives of 
fatty acids as variables, which previously were found ${ }^{26}$ to affect polyplex characteristics such as siRNA binding and lytic potential of oligomers, gene silencing efficacy and toxicity of polyplexes. Compared with their nonreducible lipo-oligomer analogs, the favorable polyplex characteristics should remain indifferent until intracellular release into the cytosol, where improved siRNA release and biocompatibility would be expected.

\section{Experimental section}

\section{Materials}

Protected Fmoc- $\alpha$-amino acids, 2-chlorotrityl chloride resin, $N, N$-dimethylformamide (DMF), $N, N$-diisopropylethylamine (DIPEA) and trifluoroacetic acid (TFA) were purchased from Iris Biotech (Marktredewitz, Germany). Triisopropylsilane (TIS), 1-hydroxybenzotriazole (HOBt), glutathione (GSH) and Triton X-100 were purchased from Sigma-Aldrich (Munich, Germany). (Benzotriazol-1-yloxy)tripyrrolidinophosphonium hexafluorophosphate (PyBOP) and microreactors were obtained from MultiSynTech (Witten, Germany). Cell culture media, antibiotics and fetal calf serum (FCS) were purchased from Invitrogen (Karlsruhe, Germany), HEPES from Biomol GmbH (Hamburg, Germany), glucose from Merck (Darmstadt, Germany), agarose NEEO Ultra-Qualität from Carl Roth GmbH (Karlsruhe, Germany) and GelRed ${ }^{\mathrm{TM}}$ from VWR (Darmstadt, Germany). Citrate-buffered human blood was provided by Klinikum der Universität München (Munich, Germany). Cell culture $5 \times$ lysis buffer and D-luciferin sodium salt were obtained from Promega (Mannheim, Germany). Ready-touse siRNA duplexes were obtained from Axolabs GmbH (Kulmbach, Germany): eGFP-targeted siRNA (siGFP) (sense: 5'-AuAucAuGGccGAcAAGcAdTsdT-3'; antisense: 5'-UGCUUGUCGGCcAUGAuAUdTsdT-3'; small letters: 2'methoxy; s: phosphorothioate) for silencing of eGFPLuc; control siRNA (siCtrl) (sense: 5'-AuGuAuuGGccuGuAuuAGdTsdT-3'; antisense: 5'-CuAAuAcAGGCcAAuAcAUdTsdT-3'). All solvents and other reagents were purchased from Sigma-Aldrich (Munich, Germany), Iris Biotech (Marktredwitz, Germany), Merck (Darmstadt, Germany) or AppliChem (Darmstadt, Germany).

\section{Synthesis of disulfide-linker building block (ssbb)}

$15.0 \mathrm{~g}$ of cystamine dihydrochloride $(66.6 \mathrm{mmol}, 1 \mathrm{eq}$.$) were$ suspended in $150 \mathrm{~mL}$ of THF with $23.2 \mathrm{ml}$ of DIPEA (133.2 mmol, 2 eq.) and cooled down to $-80{ }^{\circ} \mathrm{C} .18 .0 \mathrm{~g}$ (53.3 mmol, 0.8 eq.) of Fmoc-OSu were dissolved in $200 \mathrm{~mL}$ of THF and added dropwise over the course of $3 \mathrm{~h}$. The reaction was stirred for additional $1 \mathrm{~h}$ at $-80^{\circ} \mathrm{C}$ and then for $1 \mathrm{~h}$ at RT. DIPEA (23.2 mL, $133.2 \mathrm{mmol}, 2$ eq.) was added and the reaction mixture was cooled to $0{ }^{\circ} \mathrm{C}$. Succinic anhydride $(12.0 \mathrm{~g}$, $119.9 \mathrm{mmol}, 1.8$ eq.) was dissolved in $150 \mathrm{~mL}$ of THF. This solution was added dropwise to the reaction mixture at $0{ }^{\circ} \mathrm{C}$ and stirred over-night. The reaction mixture was concentrated to approximately $200 \mathrm{~mL}$, mixed with $200 \mathrm{~mL}$ of DCM and was washed $5 \times$ with $0.1 \mathrm{M}$ sodium citrate buffer $(\mathrm{pH}$ 5.2). The organic phase was dried over sodium bicarbonate, concentrated and purified by dry column vacuum chromatography (DCVC) using a $n$-heptane/EtOAc gradient (starting from 1:1) to elute Fmoc-byproducts, followed by a EtOAc/MeOH gradient to isolate the product. The solvent was removed under reduced pressure to give $6.2 \mathrm{~g}$ of a white solid (13.1 mmol, 24.5\%).

\section{Oligomer synthesis}

Oligomers were synthesized using a 2-chlorotrityl resin preloaded with the first C-terminal amino acid of the respective topology (see ESI $\dagger$ ) as solid support. Artificial Fmoc-oligoamino acid Fmoc-Stp(Boc3)-OH was synthesized as described before. $^{23,34}$ Oligomers of artificial oligoamino acids were synthesized manually under standard Fmoc solid phase peptide synthesis conditions using syringe microreactors. Coupling steps were carried out using 4 eq. Fmoc-amino acid, 4 eq. HOBt, 4 eq. PyBOP and 8 eq. DIPEA in DCM-DMF $1: 1(10 \mathrm{~mL}$ $\mathrm{g}^{-1}$ resin) for $90 \mathrm{~min}$. In case of coupling Fmoc-succinoyl-cystamine no HOBt was used and only DMF was used as solvent. All couplings after Fmoc-succinoyl-cystamine were carried out without HOBt. Fmoc deprotection was accomplished by $4 \times 10 \mathrm{~min}$ with $20 \%$ piperidine in DMF (10 $\mathrm{mL} \mathrm{g}^{-1}$ resin). After each coupling and deprotection step, a washing procedure comprising $3 \times 1$ min DMF, $3 \times 1$ min DCM incubation $\left(10 \mathrm{~mL} \mathrm{~g}^{-1}\right.$ resin) and a Kaiser test ${ }^{35}$ were performed. In case of a positive result of the Kaiser test after coupling, the last coupling step was repeated. In case of a negative result after deprotection, the last deprotection step was redone. Symmetrical branching points were introduced using FmocLys(Fmoc)-OH, asymmetric branching in T-shape structures was introduced using Fmoc-Lys(Dde)-OH. Dde-deprotection was performed 30 times with a Syro Wave ${ }^{\mathrm{TM}}$ synthesizer (Biotage, Uppsala, Sweden). Hydrazine-DMF solution 1:50 was added and vortexed for $2 \mathrm{~min}$. The reaction solvent was drained and fresh solution was added again. Afterwards the resin was washed with $5 \times 1 \mathrm{~min}$ DMF and $3 \times 1 \mathrm{~min}$ DCM (10 $\mathrm{mL} \mathrm{g}^{-1}$ resin).

Finally, all oligomers were cleaved off the resin by incubation with TFA-TIS- $\mathrm{H}_{2} \mathrm{O} 95: 2.5: 2.5$ (10 $\mathrm{mL} \mathrm{g}^{-1}$ resin) for $90 \mathrm{~min}$. The cleavage solution was concentrated by flushing nitrogen and oligomers were precipitated in $40 \mathrm{~mL}$ of precooled MTBE- $n$-hexane $1: 1$. All oligomers were purified by size exclusion chromatography using an Äkta purifier system (GE Healthcare Bio-Sciences AB, Uppsala, Sweden), a Sephadex G-10 column and $10 \mathrm{mM}$ hydrochloric acid solution-acetonitrile $7: 3$ as solvent. All oligomers were lyophilized. Oligomer sequences were validated by mass spectrometry and ${ }^{1} \mathrm{H}-\mathrm{NMR}$.

\section{SiRNA polyplex formation}

siRNA and oligomer at indicated nitrogen/phosphate (N/P) ratios were diluted in separate tubes of equal volumes of $20 \mathrm{mM}$ HEPES buffered 5\% glucose pH 7.4 (HBG) each. If no other description is provided, $500 \mathrm{ng}$ siRNA were dissolved in $10 \mu \mathrm{L}$ HBG. Only protonatable nitrogens were considered in the N/P calculations. The polycation solution was added to the 
siRNA solution, mixed by rapid pipetting $5 \times$ and incubated for $40 \mathrm{~min}$ at RT.

\section{siRNA binding assays}

A $2.5 \%$ agarose gel was prepared by dissolving agarose in TBE buffer (10.8 $\mathrm{g}$ of trizma base, $5.5 \mathrm{~g}$ of boric acid, $0.75 \mathrm{~g}$ of disodium EDTA, and $1 \mathrm{~L}$ of water) and subsequent boiling. After cooling down to about $50{ }^{\circ} \mathrm{C}$, GelRed ${ }^{\mathrm{TM}}$ was added. Polyplexes were formed using the stated $\mathrm{N} / \mathrm{P}$ ratios, and placed into the sample pockets after $4 \mu \mathrm{l}$ of loading buffer (prepared from $6 \mathrm{~mL}$ of glycerine, $1.2 \mathrm{~mL}$ of $0.5 \mathrm{M}$ EDTA, $2.8 \mathrm{~mL}$ of $\mathrm{H}_{2} \mathrm{O}$, $0.02 \mathrm{~g}$ of bromophenol blue) was added. Electrophoresis was performed at $100 \mathrm{~V}$ for $40 \mathrm{~min}$.

\section{siRNA polyplexes under reducing conditions}

siRNA polyplexes were formed at $\mathrm{N} / \mathrm{P}$ ratio 20 . After polyplex incubation, $5 \mu \mathrm{L}$ of a GSH solution were added to $20 \mu \mathrm{L}$ of the polyplex solution. The GSH stock solution had a concentration of $50 \mathrm{mM}$ and $\mathrm{pH}$ was adjusted to 7.4. It was diluted to concentrations of $5 \mathrm{mM}$ and $0.5 \mathrm{mM}$. Consequently, the resulting solutions had the final concentrations $0.1 \mathrm{mM}, 1 \mathrm{mM}$ and $10 \mathrm{mM}$, respectively. HBG was used as negative control (0 mM GSH). The solutions were incubated at $37{ }^{\circ} \mathrm{C}$ for 90 min. $5 \mu \mathrm{L}$ loading buffer was added and a siRNA binding assay was performed.

\section{Erythrocyte leakage assay with or without previous reductive treatment}

Fresh, citrate-buffered human blood was washed with phosphate-buffered saline (PBS). The washed human erythrocyte suspension was centrifuged and the pellet was diluted to $5 \times 10^{7}$ erythrocytes per $\mathrm{mL}$ with $\mathrm{PBS}(\mathrm{pH} 7.4,6.5$ and 5.5). In case of GSH treatment, oligomers were incubated in $10 \mathrm{mM}$ GSH in HEPES ( $\mathrm{pH}$ adjusted to 7.4) at a concentration of $1 \mathrm{mg}$ $\mathrm{mL}^{-1}$ at $37^{\circ} \mathrm{C}$ for $90 \mathrm{~min}$. A volume of $75 \mu \mathrm{l}$ of erythrocyte suspension and $75 \mu \mathrm{L}$ of oligomer solution (previously diluted with PBS of the respective $\mathrm{pH}$ ) were added to each well of a V-bottom 96-well plate (NUNC, Denmark), resulting in a final concentration of $7.5 \mu \mathrm{M}$ oligomer per well. The plates were incubated at $37^{\circ} \mathrm{C}$ under constant shaking for $1 \mathrm{~h}$. After centrifugation, $100 \mu \mathrm{L}$ of the supernatant was analyzed for hemoglobin release at $405 \mathrm{~nm}$ wavelength using a microplate reader (Spectrafluor Plus, Tecan Austria GmbH, Grödig, Austria).

\section{Cell culture}

Mouse neuroblastoma Neuro2A/eGFPLuc cells and human prostate carcinoma DU-145/eGFPLuc cells, both stably transfected with the eGFPLuc gene, were used for gene silencing assays. Neuro2A/eGFPLuc cells were grown in Dulbecco's modified Eagle's medium (DMEM). DU-145/eGFPLuc cells were grown in RPMI-1640 medium. Both culture media were supplemented with $10 \%$ FCS, $100 \mathrm{U} \mathrm{mL}^{-1}$ penicillin and $100 \mu \mathrm{g} \mathrm{mL} \mathrm{m}^{-1}$ streptomycin. The cells were maintained in ventilated flasks in the incubators at $37{ }^{\circ} \mathrm{C}$ with $5 \% \mathrm{CO}_{2}$ in a humidified atmosphere. Cell lines were grown to $80-90 \%$ confluency and harvested.

\section{Reporter gene silencing assay}

Gene silencing assays were performed using Neuro2A/eGFPLuc or DU145/eGFPLuc cells. The siRNA against eGFP (siGFP) for silencing the eGFPLuc gene or its control sequence (siCtrl) was exploited. 5000 cells per well were seeded on 96-well plates, and medium was replaced with $80 \mu \mathrm{L}$ fresh growth medium after $24 \mathrm{~h}$. Polyplex solution (20 $\mu \mathrm{l}$, prepared as described above) at indicated $\mathrm{N} / \mathrm{P}$ ratios was added to each well and incubated for $48 \mathrm{~h}$ at $37^{\circ} \mathrm{C}$. After transfection, medium was removed and cells were treated with $100 \mu \mathrm{L}$ cell lysis buffer. Luciferase activity in the cell lysate was measured using a luciferase assay kit (Promega, Mannheim, Germany) and a Centro LB 960 plate reader luminometer (Berthold Technologies, Bad Wildbad, Germany). The experiments were performed in triplicates, and the relative light units (RLU) were presented as percentage of the luciferase gene expression obtained with HBG-treated control cells.

\section{Results and discussion}

A building block applicable for standard Fmoc solid-phase peptide synthesis requires a protected amino group and a free carboxylic acid function. The synthesis of the disulfide building block (ssbb) was carried out starting from cystamine by selective protection of one terminal amine with Fmoc in the first step avoiding change of protecting groups as was previously presented. ${ }^{36}$ To achieve reaction selectivity towards mono-functionalization, $0.8 \mathrm{eq}$. Fmoc-succinimide (Fmoc-OSu) was added dropwise to a cooled solution $(-80 \mathrm{C})$ of cystamine dihydrochloride in THF with DIPEA as a base. The carboxylic acid function was introduced in the second step by addition of 1.8 eq. succinic anhydride (Succ anhydride) solved in THF with DIPEA (Fig. 1A). The ssbb structure was purified via
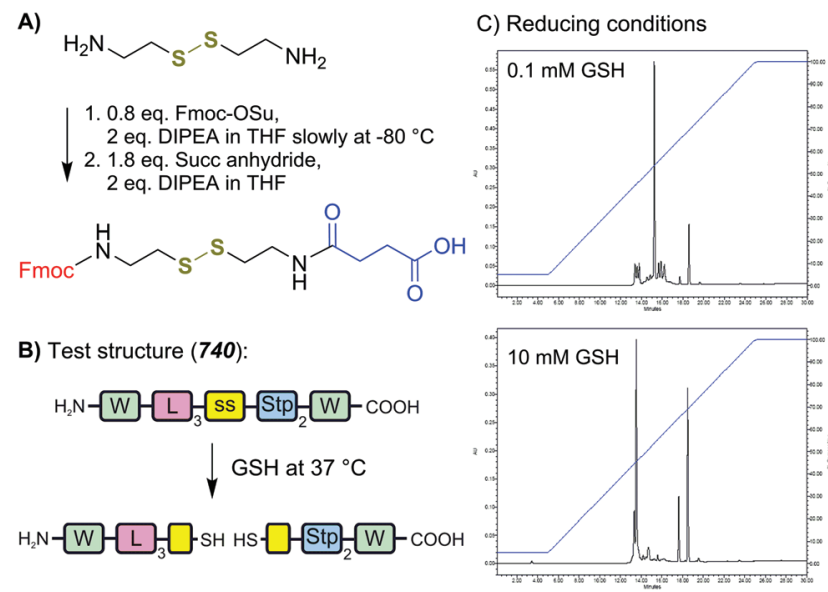

Fig. 1 (A) Synthesis of the disulfide building block Fmoc-succinoylcystamine (Fmoc-ssbb). (B) Gluthathione (GSH) triggered cleavage of test structure $(740)$ with a hydrophilic $\left(\mathrm{Stp}_{2}\right)$ and a hydrophobic $\left(\mathrm{L}_{3}\right)$ part connected by ssbb. (C) Test structure monitored by HPLC $(280 \mathrm{~nm}$ wavelength) after incubation for $90 \mathrm{~min}$ at $37^{\circ} \mathrm{C}$ in $0.1 \mathrm{mM} \mathrm{GSH}$ - (top) and $10 \mathrm{mM} \mathrm{GSH}$ - (bottom) containing HEPES buffer $\mathrm{pH}$ 7.4. 
column chromatography and the identity confirmed by ${ }^{1} \mathrm{H}$-NMR. A test structure (740) was synthesized on solid phase to prove the applicability for SPS. Here the ssbb connects a lipophilic peptide sequence containing three leucines $\left(\mathrm{L}_{3}\right)$ to a hydrophilic sequence with two succinyl-tetraethylene pentamine (Stp) units (Fig. 1B). Tryptophane (W) was incorporated into both parts to facilitate photometric analysis. The product structure was confirmed by mass spectrometry and ${ }^{1} \mathrm{H}$-NMR. The product was incubated with increasing concentrations of the physiological antioxidant glutathione (GSH) at $37{ }^{\circ} \mathrm{C}$ to simulate the behavior of the ssbb at different extra- and intracellular milieus. A GSH concentration of $0.1 \mathrm{mM}$ mimics the barely reducing extracellular environment. As expected, the test oligomer mostly retained its structural integrity. Increasing the GSH concentration to $10 \mathrm{mM}$ resembling the cytosolic reducing conditions resulted in cleavage of the test structure without detectable fractions of intact oligomer (Fig. 1C).

Next, the ssbb unit was applied in SPS of lipo-oligomers supposed to form siRNA polyplexes that are stable in the extracellular and labile in the intracellular environment. Lipo-oligomers with three different topologies, T-shape, i-shape, and U-shape, were synthesized (Fig. 2 top and $\mathrm{S} 1 \dagger$ ). As shown in our previous work, topologies as well as specific moieties of structures may influence the biophysical and biological properties of the resulting polyplexes. ${ }^{17,24,26,27}$ For bioreducible crosslinking between oligocations, previous oligomers were designed with cysteines terminating the cationic backbone. ${ }^{24,26,27}$ Differently, in the current work the ssbb unit was positioned between the ionizable oligocationic part of the molecule and a bis (fatty acid) unit. Thus, upon reductive cleavage maximum destabilization of the polyplex on the one hand, and abolition of the membrane-active amphiphilic character on the other hand, should be achieved. The oligocation part contains several Stp units as artificial oligoamino acids for nucleic acid binding and endosomal protonation, ${ }^{23}$ and lysines (K) as branching units. Additionally, the cationic part was equipped with tyrosine trimers $\left(\mathrm{Y}_{3}\right)^{27}$ for further hydrophobic stabilization of the T-shape oligomers (Fig. 2), and with histidine blocks $\left(\mathrm{H}_{6}\right)$ for increased endosomal buffering of the i-shape oligomers. The saturated $\mathrm{C} 14$ short chain myristic acid (MyrA), the stearic acid (SteA) with the longer C18 chain, and the bulky cholanic acid (CholA) were incorporated as fatty acids for hydrophobic polyplex stabilization. For all oligomers, the corresponding non-reducible control structures lacking ssbb were synthesized. The ssbb was incorporated into various different structures to proof the concept independently of shape and other functional domains and to put this work into a broader context. The structures were analyzed with mass spectrometry and ${ }^{1} \mathrm{H}$-NMR (Fig. $3 \mathrm{~A}$ and ESI $\dagger$ ). To exclude that lipo-oligomers contain significant amounts of reduced fragments, Ellman's assay was performed to detect free thiols (Table S1†). Lack of free thiols $(<3 \%)$ indicate high integrity of the ssbb linkage.

Polyplexes were formed by mixing the cationic oligomers with siRNA, followed by 40 minutes incubation and biophysical characterization (Fig. 3 for stable and reducible CholA
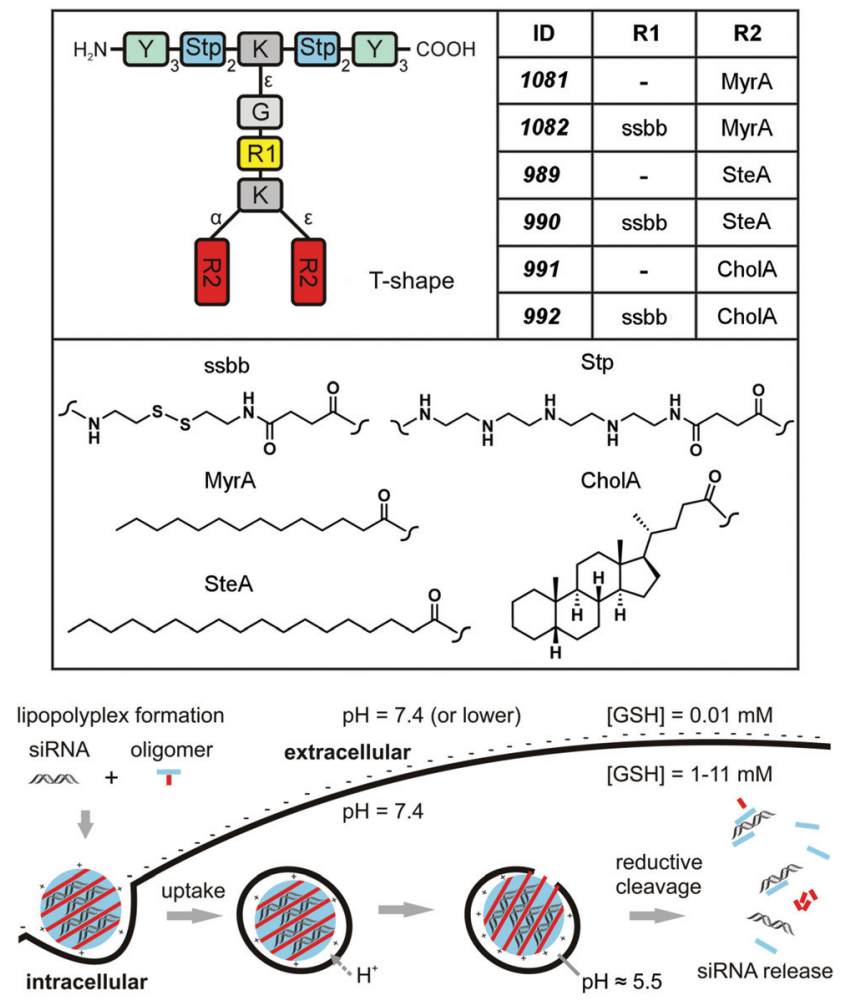

Fig. 2 Sequence-defined oligomers with T-shape topology. Top: Schematic overview of the structures with different modifications (Y: tyrosine, K: lysine, G: glycine, Stp: succinoyl-tetraethylene-pentamine, ssbb: succinoyl-cystamine, MyrA: myristic acid, SteA: stearic acid, CholA: $5 \beta$-cholanic acid). The broken lines represent amide linkages. IDs are unique database identification numbers. i-shape and U-shape structures are depicted in Fig. S1.† Bottom: Cellular uptake, acidic pH-triggered endosomal escape, and GSH triggered cytosolic disassembly of siRNA polyplexes.

T-shapes 991 and 992; see $\mathrm{ESI}^{\dagger}$ for other structures). The siRNA binding ability of oligomers was determined by measuring the electrophoretic mobility of siRNA in a $2.5 \%$ agarose gel. Different $\mathrm{N} / \mathrm{P}$ values depict the ratio of protonatable amines ( $\mathrm{N}$ ) of the oligomer to phosphates (P) of the siRNA. This does not present charge ratios, as only a fraction of the protonatable amines are protonated at physiological pH. All T-shape, i-shape and U-shape structures showed sufficient binding at N/P $\geq 12$ (Fig. 3C, S2 and S3 $\dagger$ ). Neither stable nor reducible polyplexes released free siRNA when exposed to $90 \%$ full serum at $37^{\circ} \mathrm{C}$ for two hours, indicating a high extracellular stability (Fig. S4†). In contrast, treatment of polyplexes with the physiological reducing agent $\mathrm{GSH}$ at $37{ }^{\circ} \mathrm{C}$ resulted in a dose-dependent loss of siRNA binding efficacy for the reducible but not the stable oligomers (Fig. 3C, S5 and S6†). Due to the particular position of the ssbb unit, reductive cleavage leads to the release of the lipid as the most important stabilization motif, thus keeping only a weak binding ability of the remaining cationic backbone. ${ }^{27}$ This destabilization of polyplexes is expected to provide better accessibility of siRNA at intracellular GSH concentrations $(\sim 10 \mathrm{mM})$. 
A)

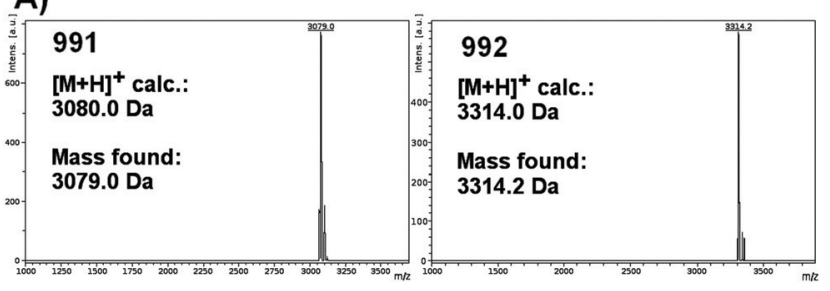

B)
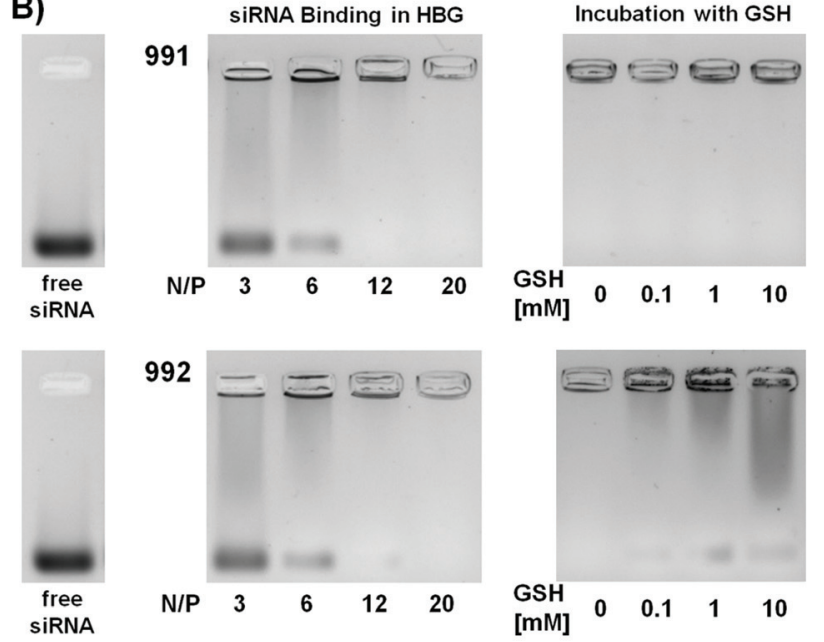

C) 991

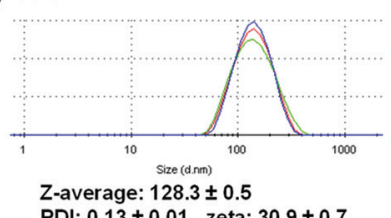

PDI: $0.13 \pm 0.01$ zeta: $30.9 \pm 0.7$

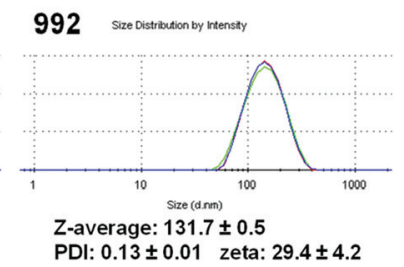

Fig. 3 (A) Analytical characterization of stable oligomer 991 and bioreducible oligomer 992 by mass spectrometry. (B) Agarose gel shift assays. Left: siRNA binding at different N/P ratios. Right: Lipopolyplexes formed at N/P 20 and subsequent 90 min treatment at $37^{\circ} \mathrm{C}$ with different concentrations of GSH in HEPES buffer pH 7.4. (C) Biophysical characterizations of lipopolyplexes formed with oligomers and siRNA at N/P 12 by DLS.

The particle sizes of siRNA lipopolyplexes were measured by dynamic light scattering (DLS). All T-shape polyplexes showed uniform sizes between 105-138 nm $z$-average (Fig. 3B and Table $\mathrm{S} 2 \dagger$ ). The sizes of i-shape and U-shape polyplexes showed higher polydispersity. All formulations revealed a positive zeta potential of around $23-32 \mathrm{mV}$ due to an excess of cationic oligomer (Table S2 $\uparrow$ ). T-shape oligomers were found as well-suited for the evaluation of structure-activity relationships, since they all formed polyplexes with reliable sizes and low polydispersity.

Gene silencing experiments were performed in Neuro2A neuroblastoma cells stably expressing an eGFP-Luciferase fusion protein (Fig. 4). Silencing by siGFP (light bars) can be quantified by a standard luciferase assay. In all cases, gene silencing was more effective for the bioreducible T-shape oligomers (Fig. 4B) as compared to their stable analogs (Fig. 4A). A reduced luciferase expression in control experiments using

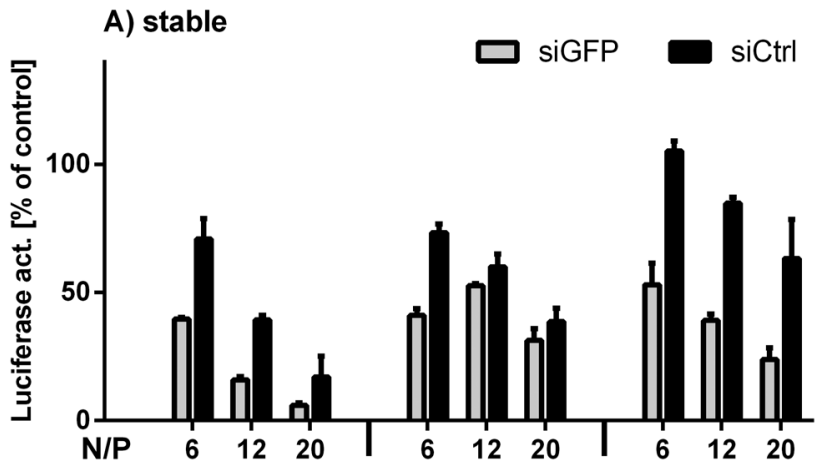

B) bioreducible (with ssbb)

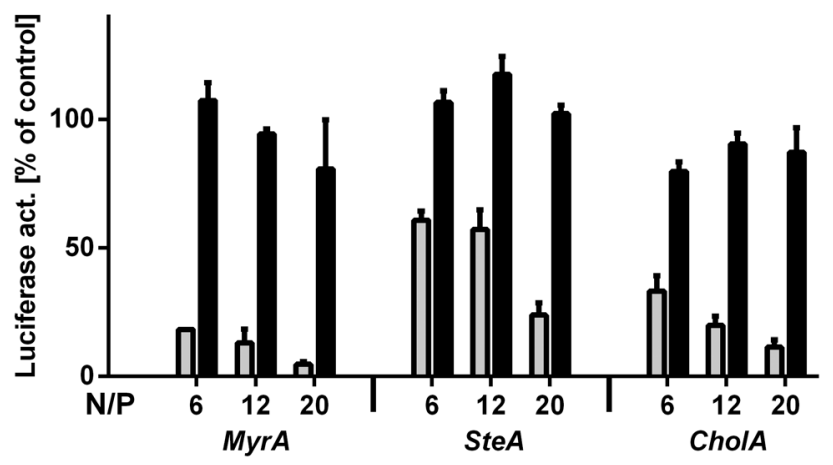

Fig. 4 Gene silencing of T-shape oligomers in neuroblastoma cells. Lipopolyplexes with $500 \mathrm{ng} / 37 \mathrm{pmol}$ eGFP-targeted siRNA (siGFP)/well respectively control siRNA (siCtrl) at N/P 6, 12 and 20 were tested for eGFPLuc gene silencing in Neuro2A-eGFPLuc cells. (A) Lipopolyplexes made of stable structures 1081, 989 and 991 (B) lipopolyplexes made of bioreducible structures 1082, 990 and 992. The luciferase activity of siRNA treated cells is presented related to buffer treated cells. HBGtreated cells were set to $100 \%$. Data are presented as mean value $( \pm S D)$ out of triplicates. Dose-dependent gene silencing transfections are shown in Fig. S8 and S9.†

siCtrl (dark bars) is caused by unspecific cytotoxic effects and not by a specific knockdown of the eGFPLuc gene. Thus, from evaluating the luciferase levels of the siCtrl polyplexes, an enhanced biocompatibility of the reducible structures (Fig. 4B) can be concluded. Similar findings, an enhanced gene silencing and especially the reduction of cytotoxicity, were also made for bioreducible i-shape and U-shape lipo-oligomers (Fig. S7 $\dagger$ ). For the best-performing T-shape structures with MyrA and CholA, dose-dependent gene silencing experiments were carried out. Based on the starting formulation of $37 \mathrm{pmol}$ siRNA (370 nM) with $1.44 \mathrm{nmol}$ oligomer (N/P 12), the dose of siRNA was reduced either at a constant N/P 12 (Fig. S8 $\dagger$ ) or a constant dose of $1.44 \mathrm{nmol}$ oligomer (Fig. S9†). In the latter case, significant gene silencing was still observed for reducible MyrA polyplexes at $1.2 \mathrm{pmol} / 12 \mathrm{nM}$ siRNA. The beneficial effects of reducible polyplexes are also confirmed in DU145/ eGFPLuc prostate cancer cells (Fig. S10†).

When focusing on the comparison of the three different fatty acids, SteA and CholA lipo-oligomers (no or only moderate silencing for the stable versions) strongly benefited from 
the incorporation of the ssbb with regard to gene silencing. In contrast, the stable MyrA lipo-oligomer displayed gene silencing activity combined with significant cytotoxicity (Fig. 4A); here the reducible ssbb unit eliminated the cytotoxicity without reducing the gene silencing activity (Fig. 4B). The findings for non-reducible lipo-oligomers are consistent with our earlier observations, ${ }^{26}$ where SteA derivatives showed poor gene silencing activity and MyrA derivatives exhibited not only gene silencing activity but cytotoxicity. For non-reducible structures, the unsaturated C18 fatty acids oleic acid and linoleic acid were the optimum lipid units with regard to transfection efficacy and cell tolerability. ${ }^{24,26,27}$ Still, due to the higher stability during synthesis and storage, in the current work saturated fatty acids were the preferred option for integration into solid phase synthesized lipo-oligomers. Nevertheless, incorporation of the bioreducible linker into oleic acid containing oligomers was also found to further enhance transfection efficacy and cell tolerability (Fig. S11†).

The different fatty acids may influence the extent of hydrophobic stabilization of siRNA polyplexes, but do also strongly affect the lytic properties of the lipo-oligomers, both in the stable and reducible setting. At endosomal $\mathrm{pH}$, the cationic

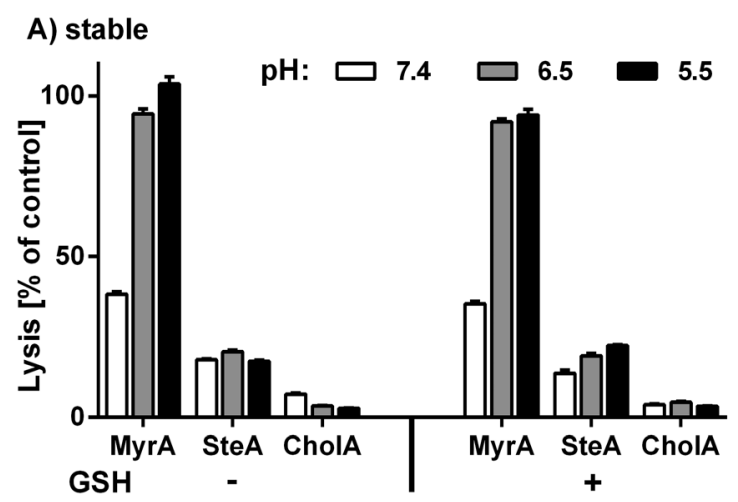

B) reducible (with ssbb)

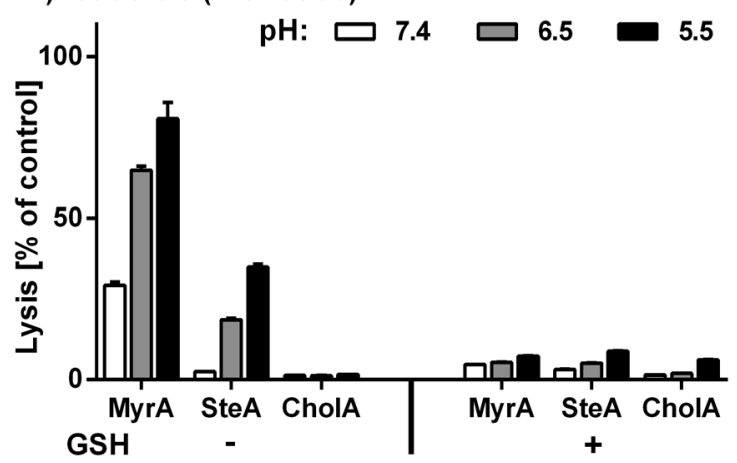

Fig. 5 Erythrocyte leakage of oligomers at different $\mathrm{pH}$ and under reducing conditions. (A) Stable lipo-oligomers 1081, 989 and 991. (B) Bioreducible lipo-oligomers 1082,990 and 992 . The final concentration of oligomers was $7.5 \mu \mathrm{M}$. GSH treated lipo-oligomers were incubated with $10 \mathrm{mM} \mathrm{GSH}$ in PBS adjusted to $\mathrm{pH} 7.4$ at $37{ }^{\circ} \mathrm{C}$ for 90 min (righthand side). PBS-treated erythrocytes were set to $0 \%$. Triton $X$ served as positive control and was set to $100 \%$. Data are presented as mean value $( \pm S D)$ out of quadruplicates. parts receive increased cationization, which in combination with the hydrophobic domain facilitates endosomal membrane destabilization and escape into the cytosol. An erythrocyte leakage assay compared the different fatty acid versions of stable (Fig. 5A) and the ssbb containing reducible (Fig. 5B) lipo-oligomers. MyrA structures displayed a far higher leakage activity (highest at $\mathrm{pH}$ 5.5) than the SteA structures, whereas oligomers with the bulky CholA did not display lytic effects. This finding is in agreement with literature, showing that cationic dimyristyl lipids strongly promote membrane fusion events. Deviations of hydrophobic volume and hydrophiliclipophilic ratio from an optimum hampered membrane interactions. $^{37}$ Treatment with GSH did not affect the stable analogs but extinguished the lytic activity of the reducible lipooligomers, consistent with their lower cytotoxicity. This observation can be attributed to the integration of the ssbb as a linker between the cationic and the lipophilic part, as reductive cleavage results in an uncharged fatty acid structure and an oligocationic part with significantly reduced amphiphilic character. Both compounds alone were not able to lyse membranes anymore.

\section{Conclusions}

We report novel lipo-oligomers as siRNA carriers with high gene silencing efficacy, which combine extracellular polyplex stability with siRNA release under cytosolic conditions, and a high lytic activity under endosomal conditions with low cytotoxicity. These apparently contradictory functional characteristics were programmed into the carriers by introduction of a bioreducible disulfide bond between the cationic backbone and the hydrophobic domain. Precise positioning was enabled by a Fmoc-protected cystamine building block compatible with solid phase assisted synthesis.

\section{Acknowledgements}

The work was supported by German Research Foundation (DFG) Excellence Cluster Nanosystems Initiative Munich (NIM), DFG SFB1032 B4 (EW), and a Research Fellowship from Bavarian Research Foundation to DJL. DP and LH thank the DFG for support through the Emmy-Noether-program (HA 5950/1-1).

\section{Notes and references}

1 T. Tuschl, ChemBioChem, 2001, 2, 239-245.

2 D. Bumcrot, M. Manoharan, V. Koteliansky and D. W. Sah, Nat. Chem. Biol., 2006, 2, 711-719.

3 E. Wagner, Expert Opin. Biol. Ther., 2007, 7, 587-593.

4 A. de Fougerolles, H. P. Vornlocher, J. Maraganore and J. Lieberman, Nat. Rev. Drug Discovery, 2007, 6, 443-453. 
5 M. E. Davis, J. E. Zuckerman, C. H. Choi, D. Seligson, A. Tolcher, C. A. Alabi, Y. Yen, J. D. Heidel and A. Ribas, Nature, 2010, 464, 1067-1070.

6 E. Wagner, Acc. Chem. Res., 2012, 45, 1005-1013.

7 Y. Sakurai, H. Hatakeyama, Y. Sato, M. Hyodo, H. Akita and H. Harashima, Mol. Ther., 2013, 21, 1195-1203.

8 S. Pinel, E. Aman, F. Erblang, J. Dietrich, B. Frisch, J. Sirman, A. Kichler, A. P. Sibler, M. Dontenwill, F. Schaffner and G. Zuber, J. Controlled Release, 2014, 182, $1-12$.

9 F. Pittella, H. Cabral, Y. Maeda, P. Mi, S. Watanabe, H. Takemoto, H. J. Kim, N. Nishiyama, K. Miyata and K. Kataoka, J. Controlled Release, 2014, 178, 18-24.

10 D. Haussecker, J. Controlled Release, 2014, 195, 49-54.

11 A. D. Tagalakis, H. D. Lee do, A. S. Bienemann, H. Zhou, M. M. Munye, L. Saraiva, D. McCarthy, Z. Du, C. A. Vink, R. Maeshima, E. A. White, K. Gustafsson and S. L. Hart, Biomaterials, 2014, 35, 8406-8415.

12 M. Mevel, T. Haudebourg, T. Colombani, P. Peuziat, L. Dallet, B. Chatin, O. Lambert, M. Berchel, T. Montier, P. A. Jaffres, P. Lehn and B. Pitard, J. Gene Med., 2016, 18, 3-15.

13 E. R. Lee, J. Marshall, C. S. Siegel, C. Jiang, N. S. Yew, M. R. Nichols, J. B. Nietupski, R. J. Ziegler, M. B. Lane, K. X. Wang, N. C. Wan, R. K. Scheule, D. J. Harris, A. E. Smith and S. H. Cheng, Hum. Gene Ther., 1996, 7, 1701-1717.

14 A. Akinc, A. Zumbuehl, M. Goldberg, E. S. Leshchiner, V. Busini, N. Hossain, S. A. Bacallado, D. N. Nguyen, J. Fuller, R. Alvarez, A. Borodovsky, T. Borland, R. Constien, A. de Fougerolles, J. R. Dorkin, J. K. Narayanannair, M. Jayaraman, M. John, V. Koteliansky, M. Manoharan, L. Nechev, J. Qin, T. Racie, D. Raitcheva, K. G. Rajeev, D. W. Sah, J. Soutschek, I. Toudjarska, H. P. Vornlocher, T. S. Zimmermann, R. Langer and D. G. Anderson, Nat. Biotechnol., 2008, 26, 561-569.

15 J. B. Ma, K. Ye and D. J. Patel, Nature, 2004, 429, 318-322.

16 L. Hartmann and H. G. Börner, Adv. Mater., 2009, 21, 34253431.

17 U. Lächelt and E. Wagner, Chem. Rev., 2015, 115, 1104311078.

18 D. L. McKenzie, K. Y. Kwok and K. G. Rice, J. Biol. Chem., 2000, 275, 9970-9977.

19 M. L. Read, S. Singh, Z. Ahmed, M. Stevenson, S. S. Briggs, D. Oupicky, L. B. Barrett, R. Spice, M. Kendall, M. Berry, J. A. Preece, A. Logan and L. W. Seymour, Nucleic Acids Res., 2005, 33, e86.
20 L. Hartmann, E. Krause, M. Antonietti and H. G. Borner, Biomacromolecules, 2006, 7, 1239-1244.

21 X. L. Wang, R. Xu, X. Wu, D. Gillespie, R. Jensen and Z. R. Lu, Mol. Pharm., 2009, 6, 738-746.

22 L. Hartmann, Macromol. Chem. Phys., 2011, 212, 8-13.

23 D. Schaffert, N. Badgujar and E. Wagner, Org. Lett., 2011, 13, 1586-1589.

24 D. Schaffert, C. Troiber, E. E. Salcher, T. Fröhlich, I. Martin, N. Badgujar, C. Dohmen, D. Edinger, R. Kläger, G. Maiwald, K. Farkasova, S. Seeber, K. Jahn-Hofmann, P. Hadwiger and E. Wagner, Angew. Chem., Int. Ed., 2011, 50, 8986-8989.

25 Q. Leng, S. T. Chou, P. V. Scaria, M. C. Woodle and A. J. Mixson, J. Gene Med., 2014, 16, 317-328.

26 T. Fröhlich, D. Edinger, R. Klager, C. Troiber, E. Salcher, N. Badgujar, I. Martin, D. Schaffert, A. Cengizeroglu, P. Hadwiger, H. P. Vornlocher and E. Wagner, J. Controlled Release, 2012, 160, 532-541.

27 C. Troiber, D. Edinger, P. Kos, L. Schreiner, R. Klager, A. Herrmann and E. Wagner, Biomaterials, 2013, 34, 16241633.

28 P. M. Klein, K. Müller, C. Gutmann, P. Kos, A. Krhac Levacic, D. Edinger, M. Höhn, J. C. Leroux, M. A. Gauthier and E. Wagner, J. Controlled Release, 2015, 205, 109-119.

29 P. M. Klein and E. Wagner, Antioxid. Redox Signaling, 2014, 21, 804-817.

30 K. L. Kozielski, S. Y. Tzeng and J. J. Green, Chem. Commun., 2013, 49, 5319-5321.

31 H. Wei, L. R. Volpatti, D. L. Sellers, D. O. Maris, I. W. Andrews, A. S. Hemphill, L. W. Chan, D. S. Chu, P. J. Horner and S. H. Pun, Angew. Chem., Int. Ed., 2013, 52, 5377-5381.

32 J. Hoon Jeong, L. V. Christensen, J. W. Yockman, Z. Zhong, J. F. Engbersen, W. Jong Kim, J. Feijen and S. Wan Kim, Biomaterials, 2007, 28, 1912-1917.

33 L. Hartmann, S. Häfele, R. Peschka-Süss, M. Antonietti and H. G. Börner, Macromolecules, 2007, 40, 7771-7776.

34 E. E. Salcher, P. Kos, T. Fröhlich, N. Badgujar, M. Scheible and E. Wagner, J. Controlled Release, 2012, 164, 380-386.

35 E. Kaiser, R. L. Colescott, C. D. Bossinger and P. I. Cook, Anal. Biochem., 1970, 34, 595-598.

36 S. Wieczorek, S. Vigne, T. Masini, D. Ponader, L. Hartmann, A. K. Hirsch and H. G. Börner, Macromol. Biosci., 2015, 15, 82-89.

37 R. Koynova, B. Tenchov, L. Wang and R. C. Macdonald, Mol. Pharm., 2009, 6, 951-958. 